\title{
Consumers' role in mental health care: a research perspective
}

\author{
A. Barbato*, Guest Editor and B. D'Avanzo, Guest Editor
}

First published online 8 June 2016

Key words: Consumers, mental health services, outcome research, peer support.

Guidelines by scientific associations and government policies in Europe, the USA and other countries over the past 30 years have promoted consumer participation in various aspects of mental health care. Although this can be viewed as an aspect of a widespread trend towards the public involvement in all sectors of healthcare (Brett et al. 2014), the specific characteristics of mental health consumers should not be overlooked, because their involvement is a challenging endeavour for a number of reasons: the power imbalance in relationships with providers is greater than in other sectors, people with mental disorders often belong to marginal groups and experience social exclusion, stigma and discrimination. Moreover, stigmatising attitudes can be found not only in general population, but also among health professionals (Nordt et al. 2006).

The growth of consumer-run services and consumer-led research represents a step forward and raises a number of key questions. Early models of consumer-run services arose in the wake of dissatisfaction with professional services and were based on the principles of exchange of experiences and mutual support. However, to what extent such services were able to meet the consumer expectations and to foster recovery more effectively than the traditional ones remained an open issue. Actually, the landmark Consumer Operated Service Programs study found modest differences in empowerment for people attending consumer-operated services in comparison with people attending mainstream services (Rogers et al. 2007). Should we conclude that the promise of alternative services has not been fulfilled? The editorial by Segal and Hayes in this issue of Epidemiology and Psychiatric Sciences addresses this problem (Segal \& Hayes, 2016).

* Address for correspondence: Dr A. Barbato, Unit for Quality of Care and Rights Promotion in Mental Health; IRCCS Istituto di Ricerche Farmacologiche Mario Negri, Via La Masa 19, 20156 Milan, Italy.

(Email: angelo.barbato@marionegri.it)
First of all, the authors point out a difference often neglected in scientific literature: consumer-operated service is an umbrella term covering a variety of organisations, not necessarily sharing the principles of selfhelp and peer support. In other words, not all consumerrun services are empowering agencies. In fact, such services can reproduce the hierarchical organization and the sharp distinction between users and providers considered as negative features of psychiatric care. Second, the authors call for a research agenda based on a careful description of the goals of consumer-run services, by looking at the content and the conceptual base of their activities. This is especially relevant, because the considerable increase in the number of consumer-run organisations has been coupled by an increase in their heterogeneity, resulting in large differences.

Consumer-operated services run the risk of becoming a source of cheap care for systems plagued by financial constraints and disappointing people looking for alternatives to mainstream care. The authors suggest that if consumer organisations are to retain a role as component of mental health care, they must keep a distinct profile, without losing their original mission. They conclude that assessment of fidelity to power sharing between staff and non-staff members, and focus on self-help should be used to describe consumer-run services and to evaluate their outcomes in terms of empowerment.

It is interesting to compare this perspective with the approach presented by Bramesfeld and Stegbauer in the second editorial of the same issue of Epidemiology and Psychiatric Sciences (Bramesfeld \& Stegbauer, 2016). The authors support the concept of responsiveness as a central feature identified by users for quality of mental health services. It pertains to a non-medical dimension of any type of services and is therefore a universal characteristic, which applies to all human organisations. It claims for (apparently) very simple and primary things, such as respect, dignity and consideration for users' values. Given the peculiar vulnerability of the population of mental health service users, these objectives seem more important and difficult to achieve than in other health services. 
This non-psychiatric specific meaningfulness of indicators is connected to the difficulty in identifying hard outcome indicators, raised by Bramesfeld and Stegbauer. Indeed, consumer assessment of outcomes either in relationship to specific interventions and to quality of service performance is rare. As a possible reason for this, the authors mention the soft nature of parameters indicated by consumers and the difficulties to translate them into indicators to be used in quantitative studies. In the few studies of quality assessment conducted from the consumers' standpoint, key indicators identified by consumers fall within the framework of responsiveness (Barbato et al. 2014). This is a serious challenge, still there are experiences where this has been tried and we should more seriously start with this. Segal \& Hayes (2016) have shown in this issue that research can be built on consumer defined constructs.

Bramesfeld \& Stegbauer (2016) warn that if we do not address the lack of representation of users' views, this lack will continue to inform guidelines and shape quality monitoring, thus perpetuating quality standard not centred on consumers and, even more serious, eventually confirming that inclusion of their views is no worth. They suggest four ways to overcome this: use of key outcomes representing patient experiences (an example can be, again, empowerment, which is a core component of the experience of care of all individuals); a more convinced acceptance and appreciation of evidence from qualitative studies; focus on the patients' pathways, i.e., the true relationship users have with services and professionals; use not only of surveys consisting of questions, but also more observational approaches, where facts, interactions, behaviour and procedures are observed and assessed according to their responsiveness.

We are persuaded that the way of evaluating service quality can change and improve through the four routes suggested here. Bramesfeld and Stegbauer also make a point of the systematic approach to quality of services evaluation. A more systematic framework for service evaluation and benchmarking would allow not only the full inclusion of users, but it would also define rules for their participation and the weight their participation should have in all the phases of the evaluation and improvement process. Active involvement of users has been often jeopardized by the initiative being in the hands of professionals who also establish participants' roles and boundaries in the evaluation process often even without making it explicit. Hence, we would need rules - to be shared - which have the responsiveness criteria integrated first of all in the co-produced service evaluation.

There are growing awareness of and sensitivity to these issues in research and several programs and initiatives are informing their own activities to the inclusion of consumers' standpoint in evidence production. Whereas the GRADE model (Barbui et al. 2010) is aimed at integrating values and human rights into the process of translation of evidence into recommendations of mental health care, the WHO QualityRights project offers an assessment and improvement tool focused on human rights conditions in mental health and social care (WHO, 2012).

The constructs of empowerment and responsiveness should be used as outcome indicators in research in mental health services as crucial aspects of care. If we fail to systematically integrate these indicators into empirical research, the gap between evidence-based medicine and consumers' experience is set to grow.

\section{Acknowledgements}

None.

\section{Financial Support}

The authors received no specific grant for this research from any funding commercial or not-for profit agency.

\section{Conflict of Interest}

None.

\section{References}

Barbato A, Bajoni A, Rapisarda F, D'Anza V, Inglese C, De Luca F, Iapichino S, Mauriello F, D'Avanzo B (2014). Quality assessment of mental health care by people with severe mental disorders: a participatory research project. Community Mental Health Journal 50, 402-408.

Barbui C, Dua T, van Ommeren M, Yasamy MT, Fleischmann A, Clark N, Thornicroft G, Hill S, Saxena S (2010). Challenges in developing evidence-based recommendations using the GRADE approach: the case of mental, neurological, and substance use disorders. PLoS Med 7, e1000322.

Bramesfeld A, Stegbauer C (2016). Assessing the performance of mental health service facilities for meeting patient priorities and health service responsiveness. Epidemiology and Psychiatric Sciences. http://dx.doi.org/10. 1017/S2045796016000354

Brett J, Staniszewska S, Mockford C, Herron-Marx S, Hughes J, Tysall C, Suleman R (2014). Mapping the impact of patient and public involvement on health and social care research: a systematic review. Health Expectations 17, 637-650.

Nordt C, Rössler W, Lauber C (2006). Attitudes of mental health professionals toward people with 
schizophrenia and major depression. Schizophrenia Bulletin 32, 709-714.

Rogers ES, Teague GB, Lichenstein C, Campbell J, Lyass A, Chen R, Banks S (2007). Effects of participation in consumer-operated service programs on both personal and organizationally mediated empowerment: results of multisite study. Journal of Rehabilitation Research and Development 44, 785-799.
Segal SP, Hayes SL (2016). Consumer-run services research and implications for mental health care. Epidemiology and Psychiatric Sciences. http://dx.doi.org/10.1017/S20457960 16000287

WHO (2012). WHO QualityRights Tool Kit to Assess and Improve Quality and Human Rights in Mental Health and Social care Facilities. World Health Organization: Geneva. 